\title{
Surface Phases and Surface Freezing in an lonic Liquid
}

Diego Pontoni, ${ }^{\dagger, \#}$ Julia Haddad,,$\#$ Bridget M. Murphy, ${ }^{\mathbf{q}, \S}$ Sven Festersen, Oleg Konovalov, $\|$ Benjamin M. Ocko, ${ }^{\perp}$ and Moshe Deutsch*, ${ }^{*}$

$\dagger$ ESRF - The European Synchrotron \&3 Partnership for Soft Condensed Matter (PSCM), 71 Avenue des Martyrs, 38000 Grenoble, France

$\ddagger$ Physics Dept. 83 Institute of Nanotechnology and Advanced Materials, Bar-Ilan University, Ramat Gan, Israel

IInstitute for Experimental and Applied Physics, Christian-Albrechts-Universität, Kiel, Germany

$\S$ Ruprecht Haensel Laboratory, Christian-Albrechts-Universität, Kiel, Germany |IESRF - The European Synchrotron, 71 Avenue des Martyrs, 38000 Grenoble, France $\perp$ NSLS-II, Brookhaven National Laboratory, Upton, NY 11973, USA

\#Contributed equally to this work

E-mail: deutsch@mail.biu.ac.il 


\begin{abstract}
Room temperature ionic liquids (RTILs), a novel class of liquid salts, are intensively studied for their basic science and numerous emerging applications. When undercooled, RTILs comprising long alkyl chains often exhibit liquid crystal (LC) bulk phases. However, only one molecular-resolution experimental structure study was published for their LC surface phases. We measured the temperature evolution of another LC surface phase, using surface specific Å-resolution x-ray methods. This phase's existence range, $90^{\circ} \mathrm{C}$, much exceeds the corresponding bulk phase's $3^{\circ} \mathrm{C}$. Its thickness, $L$, confirms the theory-predicted logarithmic temperature dependence, with an amplitude equalling the bulk correlation length. Surprisingly, at L's divergence temperature, a $\sim 20 \AA$-thick, hexagonally-packed, crystalline monolayer forms at, and fully covers, the sample's surface. It is identified as a surface-frozen Langmuir-Gibbs film, and fundamentally differs from the only reported RTIL surface crystal, a Coulomb-dominated, four-layer, island phase, covering only $5 \%-15 \%$ of the surface.
\end{abstract}




\section{Introduction}

RTIL are molten salts, comprising an organic cation with one or more alkyl chains, and a (usually smaller) organic or inorganic anion. The ions' bulky and irregular shape inhibits their solidification down to room temperatures. Short-chain RTILs, having relatively high ion mobilities, have been intensively studied for electrochemical applications in batteries, supercapacitors, solar cells, etc. ${ }^{1-4}$ Tribological RTIL studies ${ }^{5}$ reveal their electrotunable lubricity ${ }^{6}$ and quantized friction in nano-thin films. ${ }^{7}$ For long-chain RTILs, like those studied here, the recently-emerged and explosively-growing field of surface active ionic liquids (SAILs), employs the long-chain-cation's amphiphilicity in medical and pharmaceutical applications ${ }^{4,8,9}$, e.g. for suppressing and dissolving Alzheimer- and Parkinson-causing amyloids ${ }^{10}$, in micellar catalysis and synthesis $^{11}$, in enhanced oil recovery $(\mathrm{EOR})^{12}$, and more. In all these applications, and in particular in SAILs, surfaces and interfaces play an important role, rendering the elucidation of the RTILs' molecular-level organization and structure at interfaces, the subject of the present study, of prime importance and broad impact.

RTILs and their surfaces are of great importance and interest for fundamental science as well. Unlike Coulomb-dominated molten inorganic salts, RTILs comprise a near unique array of intermolecular interactions, including van der Waals (vdW), Coulomb, $\pi-\pi$, dipole, solvophobic, and hydrogen bonding. ${ }^{13,14}$ Also unlike molten inorganic salts, the RTIL's molecular structure allows tuning the relative strengths of the two main interactions, Coulomb and vdW, by varying the cation's alkyl chain length, $n .{ }^{15-17}$ Typically, for $n \leq 4-6$ the dominant bulk interaction is mostly Coulomb. Increasing $n$, renders vdW increasingly stronger, driving the bulk from a locally isotropic, homogeneous and unstructured liquid, to a locally structured one, where apolar chains segregate from the charged moieties into domains correlated over $n$-increasing nanoscale lengths. ${ }^{15,16}$ Still-longer chains induce a bulk LC phase, with the $n$-threshold for the LC phase in a given homologous cation series depending on the anion. ${ }^{18-20}$ While this bulk phase sequence has been well studied experimentally (calorimetry, x-ray diffraction, etc.) for several RTIL series ${ }^{14,21,22}$, that of the RTIL/air surface has 
been much less studied with sub-molecular resolution ${ }^{23-28}$, since the required surface specific x-ray measurements are rather challenging, and require special setups. ${ }^{29-31}$ In fact, only a single x-ray study of a LC surface phase of an RTIL, $\left[\mathrm{C}_{22} \operatorname{mim}\right]\left[\mathrm{NTf}_{2}\right]$, has been published to date, identifying the phase as smectic A, and determining the temperature dependence of the thickness of the LC surface layer. ${ }^{32}$

Here we report an x-ray study of the liquid/air surface of another long-chain RTIL, $\left[\mathrm{C}_{20} \mathrm{mim}\right]\left[\mathrm{NTf}_{2}\right]$, and its evolution with temperature upon cooling from a conventional surfacelayering phase $\mathrm{e}^{27,28}$, through the emergence and growth of a LC surface phase, to the eventual divergence of this LC surface layer's thickness to engulf the sample's bulk. Moreover, unlike $\left[\mathrm{C}_{22} \mathrm{mim}\right]\left[\mathrm{NTf}_{2}\right]$, a surface-frozen ( $\mathrm{SF}$ ) crystalline monolayer is found to appear at the RTIL/air surface upon formation of the bulk LC phase. Crystalline surface order in an RTIL has been hitherto reported only for a single Coulomb-dominated short-chain compound ${ }^{33}$, where crystalline "islands" were found to coexist at the surface with the surrounding liquid surface "sea". The islands consisted of stacks of four monomolecular layers having a rectangular lateral molecular packing. The crystalline surface monolayer found here fully covers the surface, is vdW-dominated, hexagonally-ordered, and closely resembles, albeit with distinct differences, the SF monolayer on alkane melts. ${ }^{34}$ The details and temperature dependence of the LC surface phase, and those of the SF monolayer, are presented and the underlying molecular-scale physics is discussed.

\section{Experiment}

\section{Materials}

The samples studied were 1-alkyl-3-methylimidazolium bis(trifluoromethylsulfonyl)imides, (denoted $\left[\mathrm{C}_{n} \mathrm{mim}\right]^{+}\left[\mathrm{NTf}_{2}\right]^{-}$), where $n=18,20$, purchased as powders at room temperature from Iolitec, Germany, with manufacturer-stated purities $>98 \%(n=18)$ and $>95 \%$ $(n=20)$. For further details see Supplementary Information (SI). 


\section{x-ray methods}

Since the x-ray methods used are well-documented ${ }^{34-36}$, we present here only a brief summary. Further details are given in the SI.

X-ray reflectivity: The laterally-averaged surface-normal electron density profile, $\rho(z)$, where $z$ is the surface-normal coordinate pointing into the sample, is obtained from the measured x-ray reflectivity (XRR). ${ }^{34-36} \mathrm{XRR}$ is the surface-reflected intensity fraction $R\left(q_{z}\right)$ of an x-ray beam impinging on the free surface of the sample at a grazing angle $\alpha . q_{z}=$ $(4 \pi / \lambda) \sin (\alpha)$ is the surface-normal scattering vector and $\lambda$ - the x-rays' wavelength. Using physical considerations, a mathematical model of $\rho(z)$ is constructed and its calculated XRR curve is least-squares computer-fitted to $R\left(q_{z}\right)$ to yield the model-defining parameters. ${ }^{34-36}$ Here we employ the model of Ref. 32 (see details in SI), depicted schematically in Fig. 1: coming in along the $z$ axis from the RTIL/air free surface at $z=0$, this model comprises first a layered smectic LC surface phase, extending from $z=0$ to $z=L$ (Fig. 1(a,b)). From $z=L$ down lies a "normal" layered phase (Fig. 1(a,d)), with the layering decaying

exponentially with $z$, as we found for non-LC, shorter-chain, RTILs. ${ }^{27,28}$ Both phases were assumed to have a common layer spacing $d .{ }^{32}$

Grazing incidence diffraction and Bragg rods The lateral order within the sample's surface layer was explored by Grazing Incidence Diffraction (GID). ${ }^{34-36}$ Here a verticallyaligned linear detector is rotated horizontally by an angle $\theta$ away from the x-ray reflection plane $\left(\theta=0^{\circ}\right)$. Each pixel along the detector corresponds to scattering vector components $q_{z}=(2 \pi / \lambda)(\sin \alpha+\sin \beta)$ and $q_{x}=(2 \pi / \lambda) \sin \theta \cos \beta$, where $\beta$ is the vertical angle from the sample plane of an x-ray scattered into the pixel, and $\lambda=0.5586 \AA$. Thus, a GID scan produces a scattered intensity map in the $\left(q_{x}, q_{z}\right)$ plane. The map yields two conventional plots: the GID and the Bragg rod (BR) plots. The first is the $q_{z}$-integrated intensity vs. $q_{x}$, showing the diffraction peaks of the lateral order in the surface layer. The second is 


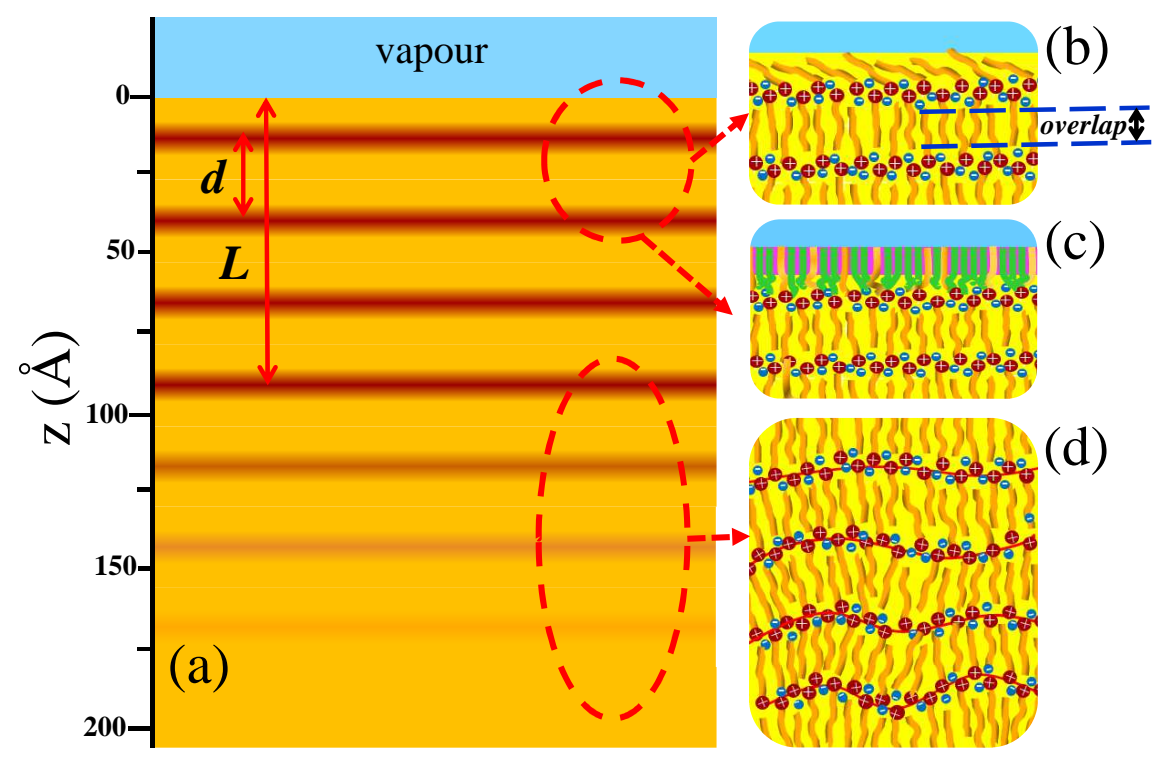

Figure 1: (a) Cartoon depicting the surface normal structure of $\left[\mathrm{C}_{20} \mathrm{mim}\right]\left[\mathrm{NTf}_{2}\right]$. Narrow brown, and broad orange, stripes represent, respectively, the charged and alkyl moieties' layers. The LC phase extends down to $z=L$, with negligible decay of the layering order. Below it lies the normal layering phase, decaying much faster with depth. $d$ is the layer spacing. (b) Near-surface layers' details at $T>53^{\circ} \mathrm{C}$, also showing the air-protruding alkyl chains of half the top-layer cations, and the chain overlap within the alkyl layer. (c) Nearsurface layers' details at $T=53^{\circ} \mathrm{C}$, showing the surface-frozen crystalline monolayer (purple slab), comprising the mixed, stretched, vertical, upper $20 \AA$ of the air-protruding cation tails (yellow) and of the alkyl-bromide guest molecules (green). (d) The $z$-decay of the "normal" layering order. The decay starts at the surface $(z=0)$ at temperatures where no LC surface phase exists, or below the LC phase where it does. The various phases and their structures are discussed in the Results and Discussion sections.

the intensity distribution along $q_{z}$ at the $q_{x}$ position of a peak in the GID plot, yielding information on the ordered layer thickness and the molecular tilt. ${ }^{34-36}$

Using for the GID scans $\alpha=0.04^{\circ}, \sim 30 \%$ below the critical angle for total external reflection $\alpha_{c}=0.056^{\circ}$, the surface-exploring beam is evanescent, its intensity decaying exponentially with depth with a $\sim 65 \AA$ decay length. ${ }^{34-36}$ Thus, it samples only the near-surface region of the RTIL ${ }^{28}$, and avoids swamping the weak surface-signal by the strong bulk-signal present for macroscopic x-ray penetration when $\alpha>\alpha_{c}$. 

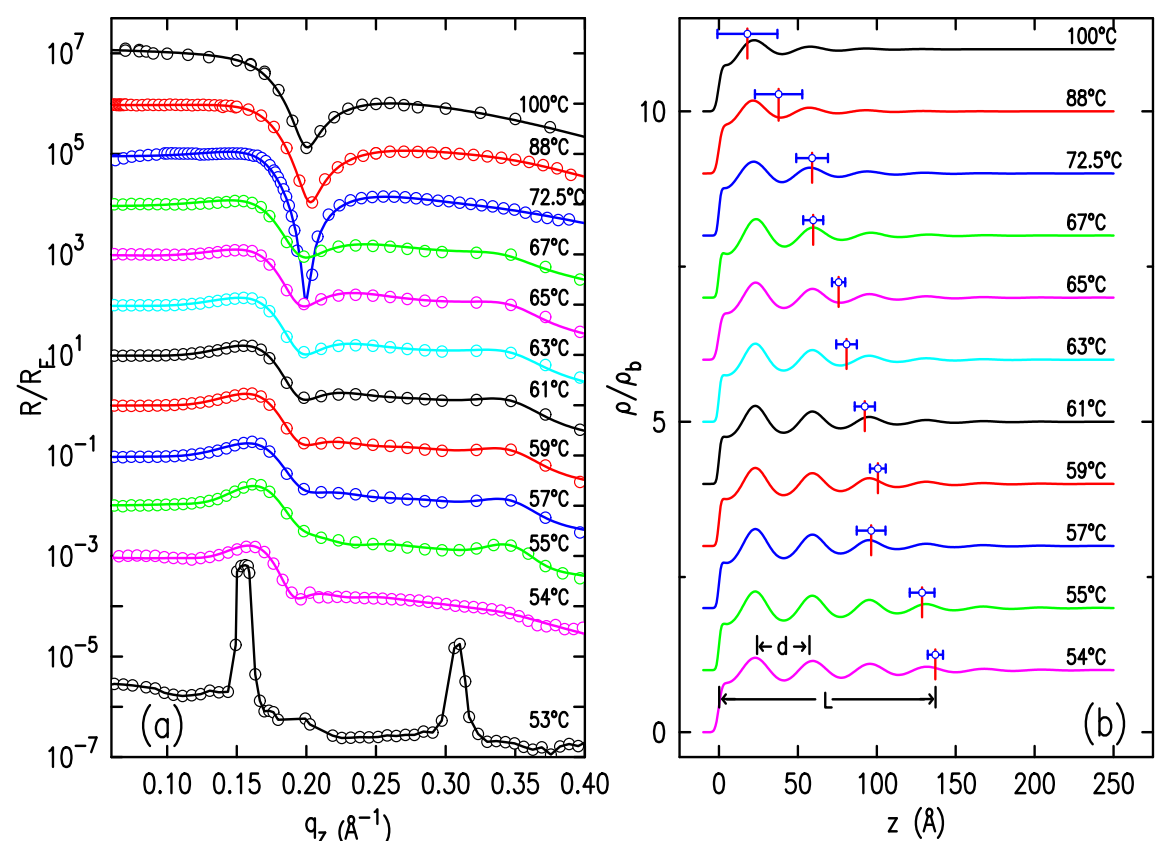

Figure 2: (a) Measured (symbols) and model fitted (lines) Fresnel-normalized x-ray reflectivity curves of $\left[\mathrm{C}_{20} \mathrm{mim}\right]\left[\mathrm{NTf}_{2}\right]$ at the temperatures listed. The line for $53^{\circ} \mathrm{C}$ is a guide to the eye only. (b) Bulk-density-normalized surface-normal electron density profiles, obtained from the model fits in (a). The red vertical lines (with their blue error bars) mark the fit-derived border $L$ between the liquid-crystal surface phase and the underlying "normal" layered RTIL phase. The layering periodicity $d$ is also marked.

\section{Results}

Fig. 2(a) presents the temperature $(T)$ evolution of the measured $R\left(q_{z}\right)$, normalized by the Fresnel XRR of an ideally smooth and abrupt surface ${ }^{35}, R_{F}\left(q_{z}\right)$, (symbols), and their model fits (lines), for $\left[\mathrm{C}_{20} \mathrm{mim}\right]\left[\mathrm{NTf}_{2}\right]$. Fig. 2(b) shows the fit-derived $\rho(z) / \rho_{b}$, where $\rho_{b}$ is the bulk electron density, and the LC layer thickness $L$. The $T \geq 72.5^{\circ} \mathrm{C}$ curves in (a) closely resemble those of $n \leq 18$ in Ref. 28, which have no LC surface phase. The single negative peak observed for $T \geq 72.5^{\circ} \mathrm{C}$ at $q_{z} \sim 0.18 \AA^{-1}$ corresponds to $d \sim 35 \AA$, in agreement with our previous studies, where the present $100{ }^{\circ} \mathrm{C}$ curve already appeared. ${ }^{15,28}$ The very thin LC surface layer at these high $T$ 's is insufficient to significantly impact the shape of $R / R_{F}$. Cooling from $T=100^{\circ} \mathrm{C}$ extends the "normal" layering deeper, as expected, rendering the negative peak sharper and larger. Below $T \leq 67^{\circ} \mathrm{C}$, phase shifts in the interfering components of the x-rays reflected from the temperature-varying stacked LC and underlying "normal" layers 
wash out the negative peak. The increasing number of layers within the LC surface phase, reflected in the increasing $L$, gradually intensifies the two orders of a LC quasi-peak, at $q_{z} \approx 0.17$ and $0.34 \AA^{-1}$. Eventually, at $T \approx 53{ }^{\circ} \mathrm{C}$ the LC surface layer's thickness diverges to engulf the bulk, yielding the sharp, intense, two orders of the LC peak observed in the bottom curve of Fig. 2(a).
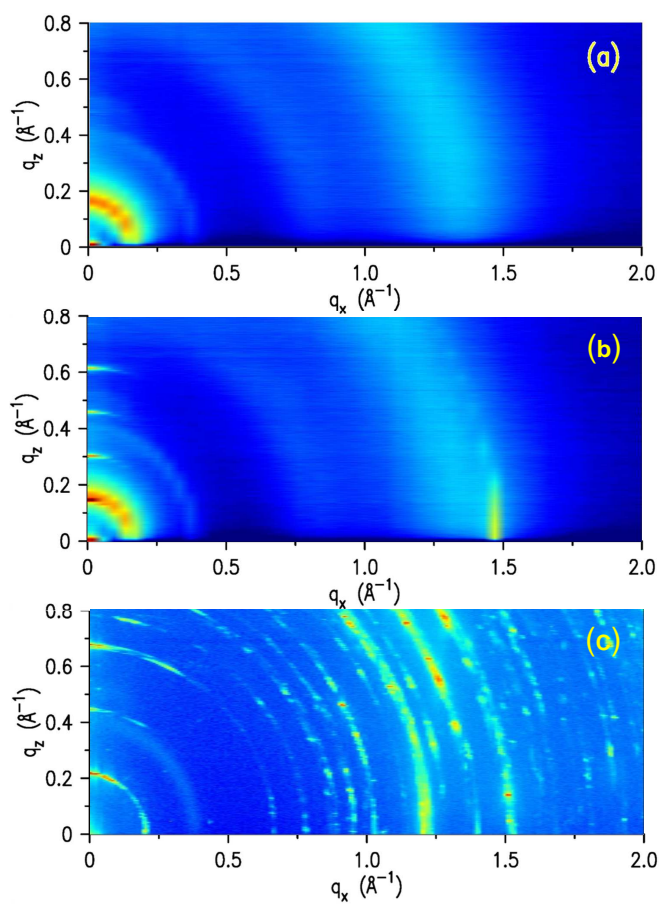

Figure 3: (a) Measured GID intensity map at $T=54{ }^{\circ} \mathrm{C}$, for $\alpha<\alpha_{c}$. The typical nanostructured RTIL liquid rings at $q_{x}^{I} \approx 0.18 \AA^{-1}, q_{x}^{I I} \approx 0.85 \AA^{-1}$ and $q_{x}^{I I I} \approx 1.38 \AA^{-1}$, are clearly visible. (b) The same map at $T=53{ }^{\circ} \mathrm{C}$, at the onset of the bulk LC phase. Additional to the liquid rings, four diffraction orders of the thick LC phase are observed along $q_{z}$. A Bragg rod is observed at $q_{x} \approx 1.49 \AA^{-1}$, indicative of an hexagonally packed, SF crystalline monolayer of extended vertically-aligned molecules.(c) The same map for $\left[\mathrm{C}_{16} \mathrm{mim}\right]\left[\mathrm{NTf}_{2}\right]$, where no LC phases exist either at the bulk or the surface. This map was measured at $T=37.5^{\circ} \mathrm{C}$, the liquid-to-crystal transition. For a discussion see text.

To search for possible lateral molecular ordering at the sample/air surface we have carried out GID measurements at the same temperatures where XRR curves were measured, using $\alpha \approx 0.7 \alpha_{c}$ as detailed in the Experimental section. The GID intensity map in Fig. 3(a), measured at $T=54^{\circ} \mathrm{C}$, shows the three main liquid RTIL rings, reported and discussed for $\left[\mathrm{C}_{20} \mathrm{mim}\right]\left[\mathrm{NTf}_{2}\right]$, and its homologous series, in our recent bulk and surface studies. ${ }^{15,28}$ The 
strong "low- $q " \operatorname{ring} I$ at $q_{x}^{I} \approx 0.18 \AA^{-1}$, is due to nanoscale segregation of apolar cationic alkyl tails from the charged moieties, causing the bulk nanostructuring ${ }^{16,17}$, and also the conventional surface layering. ${ }^{27,28}$ Rings II and III at $q_{x}^{I I} \approx 0.85 \AA^{-1}$ and $q_{x}^{I I I} \approx 1.38 \AA^{-1}$ are due to correlations of same-charge ions, i.e. the cationic headgroups and anions (II), and of intra- and inter- molecular adjacent atoms, mostly the adjacent alkyl chains (III). ${ }^{14,16,17}$ Detailed simulations support these assignments. ${ }^{37-39}$ It is important to note that in the Fig. 3(a) map no sharp structural diffraction peaks, assignable to long-range lateral order, are observed, and we conclude that no such order exists. This holds true at all measured temperatures $T>53^{\circ} \mathrm{C}$.

At $T=53^{\circ} \mathrm{C}$ (Fig. 3(b)), where the LC surface phase diverges to engulf the bulk as explained above, two new features appear, which we now discuss in some detail. The first feature is a strong, saturated, peak at $0.16-0.17 \AA^{-1}$ and its three higher-order harmonics, appearing as short horizontal "dashes" along the $q_{z}$-axis, also observed in other lamellar LC phases. ${ }^{40}$ The two lowest-order "dashes" correspond to the peaks appearing in Fig. 2(a) at $T=53{ }^{\circ} \mathrm{C}$. Since the LC phase here is smectic- $\mathrm{A}^{32}$, the appearance of an intense second-order peak is surprising, in view of its absence in conventional, non-RTIL, thermotropic smectic-A LCs. ${ }^{41-43}$ This must be due to the scatterers' form factor being non-centrosymmetric, in contrast with the centrosymmetric form-factor in conventional, non-RTIL, smectic-A LCs. ${ }^{43}$

The second, and most surprising, feature observed in Fig. 3(b) is a short, thin, and intense diffraction line at $q_{x}^{B R} \approx 1.5 \AA^{-1}$. The shape, orientation, and extension of this feature mark it as a Bragg $\operatorname{Rod}^{34,35}$, the signature of a crystalline surface monolayer of extended, surface-normal-oriented, molecules. Only a single case of a surface crystal has been hitherto reported for RTILs. However, this occurred for a short-chain, Coulomb-dominated RTIL molecule ${ }^{33}$, where the surface crystals were rectangularly-packed, 4-layered, "islands" covering only $5 \%-15 \%$ of the surface area, in coexistance with the liquid surface "sea". In contrast, the single BR observed here indicates a hexagonal packing ${ }^{34,44}$ and its absolute intensity and persistence with unchanging intensity in repeated measurements at different 
surface positions, imply that it fully covers the surface of the sample.

Finally, the fact that the LC harmonic peaks along $q_{z}$ in Fig. 3(b) form no rings but stay closely confined to the $q_{z}$ axis indicates that the LC phase, even though now macroscopically thick, preserves the surface-parallel orientation of the smectic layers, a property important for tribological applications. ${ }^{6,45}$ When RTILs freeze into a fully crystalline phase the surface and underlying bulk break up into randomly oriented crystallites, thus forming rings in the diffraction pattern. An example is shown in Fig. 3(c) for $\left[\mathrm{C}_{16} \mathrm{mim}\right]\left[\mathrm{NTf}_{2}\right]$, where the RTIL's surface and bulk freeze from a nanostructured liquid into a crystalline phase, without an intermediate LC phase.

We now proceed to discuss these experimental results in the section.

\section{Discussion}

\section{The surface-normal structure}

We discuss first the XRR results and their fits in Fig. 2 to elucidate the temperature evolution of the top LC surface phase and of the "normal" layering phase below it (see Fig. 1(a)). The LC layer thickness $L$, normalized by the layer period $d$, both derived from the fits, are plotted ( $\square$ ) in Fig. 4(a). Surface energy balance considerations ${ }^{32,46,47}$ predict that $L(T) / d=$ $\left(L_{0} / d\right) \ln \left(t_{1} / t\right)$, where $t_{1}=\left(T_{1}-T_{0}\right) / T_{0}$ and $t=\left(T-T_{0}\right) / T_{0}$, and $T_{1}$ and $T_{0}$ are the onset and the divergence temperatures of the LC surface layer, respectively. The good agreement of this expression (black dash line) with the fit-derived $L / d$ values confirms the expected logarithmic $T$-dependence, and yields $T_{0}=51.8^{\circ} \mathrm{C}$ and $T_{1}=141.8^{\circ} \mathrm{C}$, and thus a large $T_{1}-T_{0}=90^{\circ} \mathrm{C}$ existence range for the surface LC phase. The fit also yields $L_{0} / d=1.05 \pm 0.03$, which agrees well with our published $d$-normalized bulk correlation length ${ }^{15}, \xi_{b} / d=1.06 \pm 0.01$. This

close agreement confirms theoretical predictions ${ }^{46}$, and demonstrates that the same main 
correlations dominates both bulk and surface phases. ${ }^{46}$ The $L / d$ values of $\left[\mathrm{C}_{22} \mathrm{mim}\right]\left[\mathrm{NTf}_{2}\right]^{32}$ in Fig. 4(a) ( $\mathrm{O}$, and fitted red dash line) are close to ours, and yield the same $\sim 90{ }^{\circ} \mathrm{C}$ $T$-range and the same agreement of $L_{0} / d$ with $\xi_{b} / d^{32}$, albeit with a larger $L_{0} / d=1.2$ and $\xi_{b} / d=1.25-1.3^{15,22}$. The smaller value of $L_{0} / d$ of $n=20$ as compared to $L_{0} / d$ of $n=22$ reflects the eduction in the vdW interaction strength with decreasing chain length, also found for the decay lengths of conventional surface layering ${ }^{28}$ and the correlation lengths of the bulk, $\xi_{b} / d .^{15}$

While this agreement of the results for the two close- $n$ RTILs is not surprising, the presence of a LC surface phase in $\left[\mathrm{C}_{20} \operatorname{mim}\right]\left[\mathrm{NTf}_{2}\right]$ has not been hitherto reported, and implies the probable existence of a corresponding LC bulk phase. Indeed, a slow, $0.5 \mathrm{~K} / \mathrm{min}$, DSC scan, Fig. 4(b), reveals the existence of this phase, albeit over a short temperature range, $\sim 3^{\circ} \mathrm{C}$ only, and at a strong undercooling: $\sim 10^{\circ} \mathrm{C}$. The corresponding ranges for $\left[\mathrm{C}_{22} \mathrm{mim}\right]\left[\mathrm{NTf}_{2}\right]$ are $\sim 10^{\circ} \mathrm{C}$ of existence and $\sim 1^{\circ} \mathrm{C}$ of undercooling. ${ }^{32}$ It seems therefore that $\left[\mathrm{C}_{20} \mathrm{mim}\right]\left[\mathrm{NTf}_{2}\right]$ is at the lowest- $n$ limit which induces LC phases in this homologous series. We checked this hypothesis by DSC and XRR measurements on the shorter-chain homolog $\left[\mathrm{C}_{18} \mathrm{mim}\right]\left[\mathrm{NTf}_{2}\right]$. Indeed, neither bulk nor surface LC phases were detected by both techniques. The large increase in undercooling, and decrease in the existence range, incurred upon reducing the cation's chain length, $n \approx 20$, by 2 methylenes only, implies that a rather small, $10 \%$, reduction in the $\mathrm{vdW}$ interaction strength suffices to offset the interaction balance dominating the RTIL's bulk structure and eliminate the LC phase. Further insight into the $n$ and $T$ phase boundaries of the LC surface and bulk phases, and a more accurate determination of their values, may be obtained from concentration-dependent measurements on $\left[\mathrm{C}_{18} \operatorname{mim}\right]\left[\mathrm{NTf}_{2}\right] /\left[\mathrm{C}_{20} \operatorname{mim}\right]\left[\mathrm{NTf}_{2}\right]$ mixtures. For the bulk, such binary RTIL mixtures were shown to behave as a single homolog having a concentration-interpolated non-integer $n$ value, intermediate between those of the two components. ${ }^{48}$ Such measurements may also provide insight into the ways that the presence of a surface modifies the bulk's non-integer $n$.

Note also that while the good fits obtained in Fig. 2(a) with a model using the same $d$ 


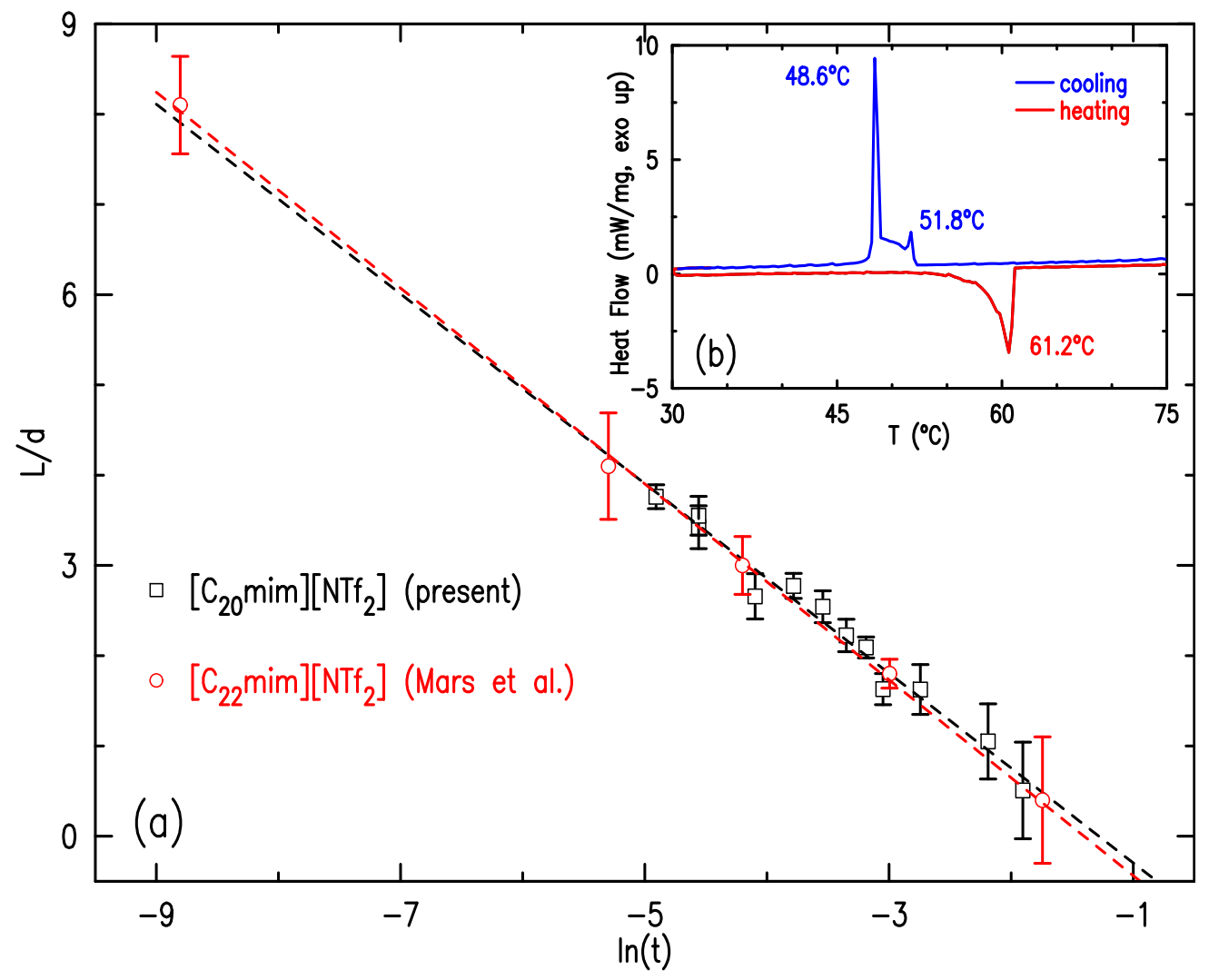

Figure 4: (a) Measured (symbols) and theory-fitted (lines) liquid-crystal surface layer thickness, $L$, normalized to the layering periodicity $d$ of the specified RTILs. Both compounds conform to the predicted logarithmic $T$-dependence, with a slight slope difference, indicating a corresponding difference in the bulk correlation length. For discussion see text. (b) Differential Scanning Calorimetry (DSC) scan $(0.5 \mathrm{~K} / \mathrm{min})$ for $\left[\mathrm{C}_{20} \mathrm{mim}\right]\left[\mathrm{NTf}_{2}\right]$, showing melting at $61.2{ }^{\circ} \mathrm{C}$, the formation of a strongly-undercooled bulk liquid-crystal phase at $51.8^{\circ} \mathrm{C}$ and the bulk freezing at $48.6{ }^{\circ} \mathrm{C}$.

for the microscopic few-monolayer-thick LC surface layer and for the underlying "normal" layered phase yield $d \approx 35.5 \AA$, the $q_{z}$ positions of the "dashes" along $q_{z}$ in Fig. 3(b), and the $T=53{ }^{\circ} \mathrm{C}$ peaks in Fig. 2(a), yield for the post-divergence LC phase a larger $d_{L C}=2 \pi / q_{z}=39 \AA$. Since the near-surface structure in the "normal" liquid phase consists of alternating thin $(\sim 5 \AA)$ layers of the charged moieties (cation headgroups and anions) and thick $(\sim 30 \AA)$ apolar layers of alkyl chains ${ }^{27,28}$, the $\sim 3.5 \AA$ higher $d_{L C}$ could result from a staggering of the headgroups in the charged moieties' layers in the macroscopic LC phase thus increasing their thickness, from an expansion of the apolar alkyl layers, or from a combination of both. A significant contribution from headgroup rearrangement is unlikely, 
since such rearrangement would change the same-charge ion distances, shifting the $q_{x}^{I I}$ ring position. Comparing Fig. 3(b) and (a), no shift in $q_{x}^{I I}$ is observed. The model of flexible, kinked, interdigitated chains proposed for the alkyl layer ${ }^{15-17,49-51}$ could, however, explain an alkyl layer expansion as resulting from an opposite-direction shift of adjacent interdigitated molecules along the layer-normal, decreasing the chains' overlap (Fig. 1(b)). Indeed, the bulk $d$ was observed to be consistently larger than the surface one also for lower- $n$ molecules in our homologous RTIL series, which do not exhibiting a LC phase, albeit with a smaller $1-2 \AA$ difference. ${ }^{15}$ An additional stretching of the flexible, kinked, chains by the increased lateral compression due to the stronger vdW interaction in the LC phase ${ }^{32}$ could perhaps also account for part of this $d$-difference.

\section{The surface-parallel structure}

The GID measurements that probe the lateral structure of the surface show in Fig. 3(b) the appearance at $T=53^{\circ} \mathrm{C}$ of a single Bragg rod at $q_{x} \approx 1.5 \AA^{-1}$. The $q_{z}$ intensity distribution of this BR, shown in Fig 5(a), peaks at $q_{z} \approx 0 \AA^{-1}$ and is $\sim 0.16 \AA^{-1}$ wide (HWHM) indicating, respectively, a monolayer of surface-normal scatterers $\sim 20 \AA$ long. The BR's integrated intensity, and its high-resolution $\left(q_{x}, q_{z}\right)$ intensity map, are plotted in Fig. 5(b) and (c), respectively. A fit by the theoretical profile ${ }^{34,35}$ (see SI) in Fig. 5(a) (line) yields a scatterer length of $\ell=19 \pm 1 \AA$, much shorter than either the layer spacing $d$ or the calculated extended length of the cations' alkyl tails ${ }^{34,52}, l_{n}=1.27 \times(n-1)+1.5=25.6 \AA$, and a tilt from the surface normal $\lesssim 2^{\circ}$. The Lorentzian fit (line) to the measured GID $q_{x}$-peak in Fig. $5(\mathrm{~b})$ yields its position, $q_{x}^{B R}=1.493 \pm 0.001 \AA^{-1}$, and FWHM, $\Delta q_{x}^{B R}=$ $0.022 \pm 0.001 \AA^{-1}$. This $q_{x}^{B R}$ indicates a (hexagonal) lattice constant $a=4 \pi /(\sqrt{3} \times 1.493)=$ $4.860 \pm 0.003 \AA$ and an area per molecule $A_{S F}=(\sqrt{3} / 2) a^{2} \approx 20.5 \AA^{2}$, typical of close-packed, extended, untilted, alkyl chains ${ }^{44}$, and very close to those of surface-frozen monolayers on 


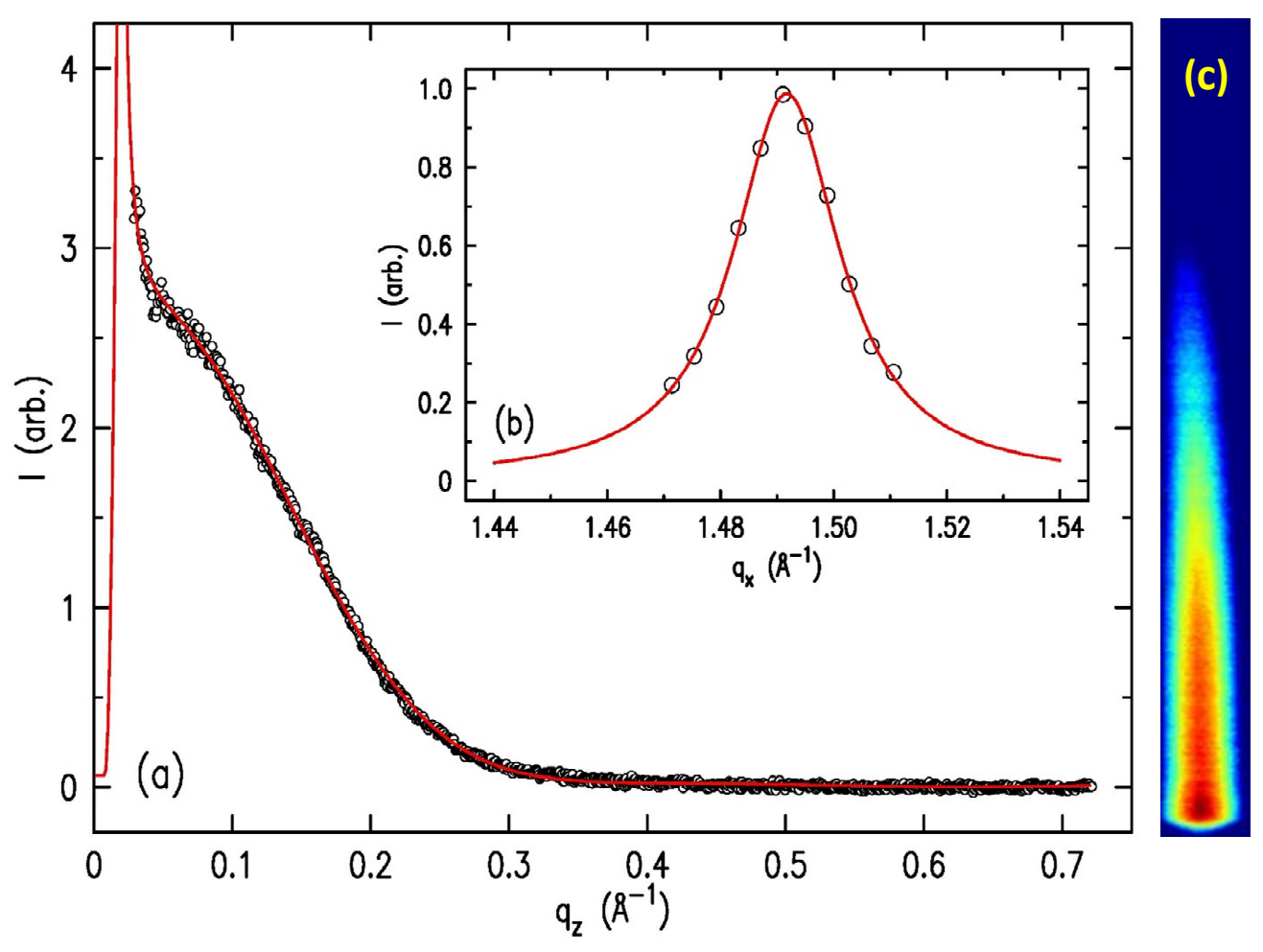

Figure 5: (a) The measured (symbols) and theory-fitted (red line) intensity distribution along the BR in Fig. 3(b). (b) The $q_{x}$-measured (symbols), and Lorentzian fitted (line), GID peak corresponding to the $\mathrm{BR}$. The position and width yield, respectively, the lattice constant $a=4.86 \AA$ and crystalline coherence length $\xi_{S F} \approx 370 \AA$. (c) A high resolution intensity map of the BR observed in Fig. 3(b), covering the $\left[q_{x}\right.$ (horizontal), $q_{z}$ (vertical)]-region from $[1.48,0] \AA^{-1}$ to $[1.505,0.25] \AA^{-1}$. See text for discussion.

alkane melts ${ }^{34}$ and of water-supported crystalline Langmuir-Gibbs (LG) monolayers. ${ }^{53-55}$

To elucidate the nature of our SF monolayer, we note that the molecular area of $\left[\mathrm{C}_{n} \operatorname{mim}\right]\left[\mathrm{NTf}_{2}\right]$ in its "normal liquid" layered surface phase ${ }^{28}, A_{R T I L} \approx 60 \AA^{2}$, is threefold larger than $A_{S F}$ above. Thus, the SF layer observed here can not consist solely of $\left[\mathrm{C}_{20} \mathrm{mim}\right]\left[\mathrm{NTf}_{2}\right]$ cationic alkyl tails, even if $100 \%$ of such tails in the topmost cation surface layer point into the vapour, instead of only $60 \%$, as found for the "normal" liquid surface phase ${ }^{28}$ and shown schematically in Fig. 1(b). Thus, we suggest that the SF monolayer is a new type of a LG film. To asses this suggestion we discuss first conventional, surfactant solution supported, LG surface films.

Conventional LG films ${ }^{53,56-58}$ form when a liquid alkane droplet, which does not spread on pure water, is placed on the free surface of an aqueous solution of a surfactant comprising 
an alkyl tail of length similar to that of the droplet's alkane. The Gibbs-adsorbed surfactant layer residing at the solution's surface induces migration of alkane molecules from the droplet along the surface, and a monolayer of mixed alkane molecules and air-protruding surfactant tails forms on the solution's free surface. Since only a single monolayer forms, and the excess alkane remains aggregated, the mixed monolayer is clearly autophobic. ${ }^{59}$ The alkane molecules intrude between the air-protruding alkyl tails of the surfactants, the lateral spacing of which is determined by the surfactant's bulk concentration. Note, however, that even at maximal surfactant surface coverage, occurring at the bulk critical micelle concentration (cmc), the surfactant tails' spacing is significantly larger than that of close-packed extended alkyl chains. ${ }^{57}$ The intruding alkanes, vertically aligned by the surfactants' air-protruding tails, thus reduce the area/chain to the canonical $A_{a l k} \approx 20 \AA^{2}$ required for forming a closepacked crystalline monolayer of alkyl chains. ${ }^{34,44}$ Indeed, while SF does not occur in the alkane-free Gibbs-adsorbed, pure surfactant, surface film, the mixed LG monolayer surfacefreezes upon cooling, forming a hexagonally-packed crystalline monolayer of surface-normalaligned mixed alkane molecules and surfactant tails.

We suggest, therefore, that the crystalline monolayer observed here is a surface-frozen LG film, where the air-protruding alkyl tails of the top cation layer of the sample (see Fig. 1(b)) play the role of the surface-adsorbed surfactants, and alkylated guest molecules, such as traces of the alkyl-bromide used in the synthesis, play the role of the alkanes. The structure of this crystalline monolayer is schematically depicted in Fig. 1(c). NMR spectroscopy (see SI) indeed confirms the presence of alkyl-bromide guest molecules in the sample at a $\sim 2$ mol\% level, consistent with the material's purity and the synthetic route followed. This concentration is much more than needed for inclusion in the single SF monolayer. These molecules remain dissolved in the "normal" liquid RTIL bulk. They are squeezed out to the sample's surface only when the more-ordered bulk LC phase forms. This would explain why the SF monolayer appears only at the onset of the LC surface phase's divergence into the bulk, at $53^{\circ} \mathrm{C}$. 
The discussion above indicates a 3-5 fold outnumbering of the cation tails by the guest molecules in the LG monolayer. Similar and even larger, >10-fold, outnumberings were found in conventional LG films at the water/air ${ }^{53,56-58}$ and water/alkane ${ }^{60}$ interfaces. Although a minority, the tail's presence in the conventional LG film was found to be mandatory for inducing $\mathrm{SF}$ in the monolayer. ${ }^{57}$ This is because in both systems the surfactant-or-cation tails promote spreading ${ }^{61,62}$ of guest chain molecules on the surface, and orient the chains side-by-side and surface-normal, thus helping the short-range intermolecular vdW attraction to form the crystalline SF monolayer. The formation in both systems of only a single surface monolayer, implying its autophobicity ${ }^{59}$, further supports the suggested structural similarity of the SF monolayer in both systems.

The lateral extent of the monolayer's crystalline order is characterized by the crystalline coherence length given by the Debye-Scherrer formula ${ }^{63} \xi_{S F}=0.9 \times 2 \pi / \sqrt{\left(\Delta q_{x}^{B R}\right)^{2}-\left(\Delta q_{x}^{\text {res }}\right)^{2}} \approx$ $370 \AA$, where $\Delta q_{x}^{\text {res }}=0.016 \AA^{-1}$ is the experimental resolution. This $\xi_{S F}$ coincides with the values obtained for water-supported LG monolayers ${ }^{53}$, in line with the assignment offered here. The thinner-than-expected monolayer, $\sim 20 \AA$ only, may result from ordering of only the top parts of the chains, due to packing frustration caused by the areal mismatch between the headgroups and the chains. Similar mismatch-inhibited partial-length ordering has been observed in amorphous- $\mathrm{SiO}_{2}$-supported monolayers of alkyltrichlorosilanes ${ }^{64}$ and alkanols ${ }^{65}$, while a nearly-matching sapphire substrate yields a full-length ordering for alkanols. ${ }^{66} \mathrm{Al}-$ though here the alkyl tails' lower ends are not firmly anchored to solid substrate sites but rather to the cation headgroups, the headgroup size provides a hard-sphere closest approach for adjacent tails' lower ends, keeping them apart by more than the chain-preferred $\sim 4.8 \AA$ as firmly as if they were substrate-anchored. Finally, while the present tentative LG-film assignment accounts for the observations, and seems therefore plausible, we can not rule out the possibility that the abrupt thickness-divergence of the LC surface layer near $53{ }^{\circ} \mathrm{C}$ somehow causes an ion rearrangement in the top surface layer such that the top $20 \AA$ of the cationic chains are brought into close-enough contacts for their vdW interaction to induce 
surface-freezing, without, or with minimal, additional guest molecules intruding between the cationic chains.

\section{Conclusions}

The present temperature-dependent x-ray and DSC study of the surface and bulk phases of $\left[\mathrm{C}_{20} \mathrm{mim}\right]\left[\mathrm{NTf}_{2}\right]$ reports for the first time the existance of liquid-crystal surface and bulk phases in this compound, and presents a number of new results, important for long-chain RTILs in general, and for their LC phases and SAILs in particular. These findings are:

- Measurement of the temperature evolution of the surface-normal structure, showing that the LC surface phases's thickness $L$ conform to the theory-predicted ${ }^{32,46}$ logarithmic temperature dependence, with an amplitude equalling the bulk correlation length, thus demonstrating that bulk correlations govern also the surface structure.

- A demonstration that the bulk LC phase forms by a surface-induced inhomogeneous nucleation ${ }^{67,68}$, through the divergence of the LC surface phase's thickness at $\sim 53{ }^{\circ} \mathrm{C}$.

- A hitherto unreported abrupt $\sim 3.5 \AA$ increase in the layer period $d$ upon transition from a "normal" to a LC bulk phase. This shift is tentatively assigned to an opposite-direction shift of adjacent interdigitated chains within the alkyl domains, thus decreasing their overlap and increasing their headgroups' layer spacing.

- A discovery of an abrupt formation of a SF crystalline monolayer at the sample's surface at the temperature where the LC surface phase diverges to engulf the bulk.

- A detailed characterization of the crystalline monolayer's structure: vdW-dominated hexagonal packing of surface-normal alkyl chains with $20.5 \AA^{2} /$ chain, and full surface coverage. The only other RTIL surface crystal reported to date ${ }^{33}$ occurs for a shortchain, Coulomb-dominated RTIL, forming 4-layered, rectangularly-packed, crystalline islands covering only $5 \%-15 \%$ of the surface. 
- Identification of the crystalline monolayer as a new type of a surface-frozen LG monolayer ${ }^{53}$, a mixture of cationic tails and alkylated guest molecules (e.g. alkyl-bromides used in the RTIL synthesis). These are squeezed out of the bulk upon formation of its LC phase, and fill the gaps between the top cation layer's air-protruding chains. All hitherto reported LG films employ aqueous surfactant solutions with surface-adsorbed surfactant monolayers. Here of course no water, or other solvent, is involved.

Similar studies on RTILs with different-size and different-shape headgroups and anions, including mixtures with alkanes and their derivatives, may provide further insight into surface freezing and surface phase behaviour in a broader range of RTILs. For example, in conventional LG films the surface density of surfactant molecules can be tuned by varying the aqueous solution's bulk surfactant concentration. In pure RTILs, however, the sizes of the anion and cation headgroups determine the surface density of cation tails. Nevertheless, this density can still be tuned by using different-size anions and cation headgroups, and cations with more than one alkyl tail. ${ }^{69}$

Finally, we note that currently-scarce molecular-resolution studies, similar to ours, of RTILS at solid and liquid interfaces, particularly of long-chain RTILs which are surface active, should provide fresh insights for both fundamental science, and electrochemical ${ }^{1,3,70}$, tribological $^{5-7}$, and medical ${ }^{4,8,9}$ applications of RTILs and SAILs.

\section{Acknowledgement}

We thank ESRF for beamtime at ID15 and ID10, ESRF's Partnership for Soft Condensed Matter (PSCM) for support, Drs. V. Honkimäki and T. Buslaps (ESRF) for assistance and advice, H. Gottlieb and M. Afri (Bar-Ilan) for NMR consultation and G. Shmul (Weizmann Institute) for HR-ESI-MS measurements and insightful discussions. We also thank PETRA III at DESY, a member of Helmohltz Association (HGF), for beamtime at P08, using the LISA instrument, funded by BMBF grants 05K13FK2 and 05K16FK1. 


\section{Supporting Information Available}

Sample, experimental, and data analysis details.

\section{References}

(1) Fedorov, M. V.; Kornyshev, A. A. Ionic Liquids at Electrified Interfaces. Chem. Rev. 2014, 114, 2978-3036.

(2) Rotenberg, B.; Salanne, M. Structural Transitions at Ionic Liquid Interfaces. J. Phys. Chem. Lett. 2015, 6, 4978-4985.

(3) Russina, O.; Lo Celso, F.; Plechkova, N. V.; Triolo, A. Emerging Evidences of Mesoscopic-Scale Complexity in Neat Ionic Liquids and Their Mixtures. J. Phys. Chem. Lett. 2017, 8, 1197-1204.

(4) Welton, T. Ionic Liquids: A Brief History. Biophys. Rev. 2018, 10, 691-706.

(5) Lhermerout, R.; Diederichs, C.; Perkin, S. Are Ionic Liquids Good Boundary Lubricants? A Molecular Perspective. Lubricants 2018, 6, 9.

(6) Fajardo, O. Y.; Bresme, F.; Kornyshev, A. A.; Urbakh, M. Electrotunable Friction with Ionic Liquid Lubricants: How Important Is the Molecular Structure of the Ions? J. Phys. Chem. Lett. 2015, 6, 3998-4004.

(7) Smith, A. M.; Lovelock, K. R. J.; Gosvami, N. N.; Welton, T.; Perkin, S. Quantized Friction Across Ionic Liquid Thin Films. Phys. Chem. Chem. Phys. 2013, 15, 1531715320.

(8) Egorova, K. S.; Gordeev, E. G.; Ananikov, V. P. Biological Activity of Ionic Liquids and Their Application in Pharmaceutics and Medicine. Chem. Rev. 2017, 117, 7132-7189. 
(9) Benedetto, A.; Galla, H.-J. Ionic Liquids and Biomolecules: Special Issue. Biophys. Rev. 2018, 10, 687-690.

(10) Takekiyo, T.; Yoshimura, Y. Suppression and Dissolution of Amyloid Aggregates Using Ionic Liquids. Biophys. Rev. 2018, 10, 853-860.

(11) Cognigni, A.; Gaertner, P.; Zirbs, R.; Peterlik, H.; Prochazka, K.; Schrder, C.; Bica, K. Surface-Active Ionic Liquids in Micellar Catalysis: Impact of Anion Selection on Reaction Rates in Nucleophilic Substitutions. Phys. Chem. Chem. Phys. 2016, 18, 1337513384.

(12) Rodriguez-Escontrela, I.; Puerto, M. C.; Miller, C. A.; Soto, A. Ionic Liquids for LowTension Oil Recovery Processes: Phase Behavior Tests. J. Coll. Interf. Sci. 2017, 504, $404-416$.

(13) Crowhurst, L.; Lancaster, N. L.; Perez Arlandis, J. M.; Welton, T. Manipulating Solute Nucleophilicity with Room Temperature Ionic Liquids. J. Am. Chem. Soc. 2004, 126, 11549-11555.

(14) Hayes, R.; Warr, G. G.; Atkin, R. Structure and Nanostructure in Ionic Liquids. Chem. Rev. 2015, 115, 6357-6426.

(15) Pontoni, D.; Haddad, J.; Di Michiel, M.; Deutsch, M. Self-Segregated Nanostructure in Room Temperature Ionic Liquids. Soft Matter 2017, 13, 6947-6955.

(16) Martinelli, A.; Marechal, M.; Ostlund, A.; Cambedouzou, J. Insights Into the Interplay Between Molecular Structure and Diffusional Motion in 1-Alkyl-3-Methylimidazolium Ionic Liquids: A Combined PFG NMR and X-ray Scattering Study. Phys. Chem. Chem. Phys. 2013, 15, 5510-5517.

(17) Russina, O.; Triolo, A.; Gontrani, L.; Caminiti, R. Mesoscopic Structural Heterogeneities in Room-Temperature Ionic Liquids. J. Phys. Chem. Lett. 2012, 3, 27-33. 
(18) Bradley, A. E.; Hardacre, C.; Holbrey, J. D.; Johnston, S.; McMath, S. E. J.; Nieuwenhuyzen, M. Small-Angle X-ray Scattering Studies of Liquid Crystalline 1-Alkyl-3methylimidazolium Salts. Chem. Mat. 2002, 14, 629-635.

(19) Greaves, T. L.; Broomhall, H.; Weerawardena, A.; Osborne, D. A.; Canonge, B. A.; Drummond, C. J. How Ionic Species Structure Influences Phase Structure and Transitions From Protic Ionic Liquids to Liquid Crystals to Crystals. Faraday Discuss. 2018, 206, 29-48.

(20) Goossens, K.; Lava, K.; Bielawski, C. W.; Binnemans, K. Ionic Liquid Crystals: Versatile Materials. Chem. Rev. 2016, 116, 4643-4807.

(21) Li, S.; Banuelos, J. L.; Guo, J.; Anovitz, L.; Rother, G.; Shaw, R. W.; Hillesheim, P. C.; Dai, S.; Baker, G. A.; Cummings, P. T. Alkyl Chain Length and Temperature Effects on Structural Properties of Pyrrolidinium-Based Ionic Liquids: A Combined Atomistic Simulation and Small-Angle X-ray Scattering Study. J. Phys. Chem. Lett. 2012, 3, $125-130$.

(22) Weiss, H.; Mars, J.; Li, H.; Kircher, G.; Ivanova, O.; Feoktystov, A.; Soltwedel, O.; Bier, M.; Mezger, M. Mesoscopic Correlation Functions in Heterogeneous Ionic Liquids. J. Phys. Chem. B 2017, 121, 620-629.

(23) Jeon, Y.; Sung, J.; Bu, W.; Vaknin, D.; Ouchi, Y.; Kim, D. Interfacial Restructuring of Ionic Liquids Determined by Sum-Frequency Generation Spectroscopy and X-Ray Reflectivity. J. Phys. Chem. C 2008, 112, 19649-19654.

(24) Sloutskin, E.; Ocko, B.; Tamam, L.; Kuzmenko, I.; Gog, T.; Deutsch, M. Surface Layering in Ionic Liquids: An X-ray Reflectivity Study. J. Am. Chem. Soc. 2005, 127, $7796-7804$.

(25) Nishi, N.; Uruga, T.; Tanida, H.; Kakiuchi, T. Temperature Dependence of Multilay- 
ering at the Free Surface of Ionic Liquids Probed by X-ray Reflectivity Measurements. Langmuir 2011, 27, 7531-7536.

(26) Wakeham, D.; Nelson, A.; Warr, G. G.; Atkin, R. Probing the Protic Ionic Liquid Surface Using X-ray Reflectivity. Phys. Chem. Chem. Phys. 2011, 13, 20828-20835.

(27) Mezger, M.; Ocko, B. M.; Reichert, H.; Deutsch, M. Surface Layering and Melting in an Ionic Liquid Studied by Resonant Soft X-ray Reflectivity. Proc. Natl. Acad. Sci. U. S. A. 2013, 110, 3733-3737.

(28) Haddad, J.; Pontoni, D.; Murphy, B. M.; Festersen, S.; Runge, B.; Magnussen, O. M.; Steinrück, H.-G.; Reichert, H.; Ocko, B. M.; Deutsch, M. Surface Structure Evolution in a Homologous Series of Ionic Liquids. Proc. Natl. Acad. Sci. U. S. A. 2018, 115, E1100-E1107.

(29) Honkimäki, V.; Reichert, H.; Okasinski, J. S.; Dosch, H. X-Ray Optics for Liquid Surface/Interface Spectrometers. J. Synch. Rad. 2006, 13, 426-431.

(30) Murphy, B. M.; Greve, M.; Runge, B.; Koops, C. T.; Elsen, A.; Stettner, J.; Seeck, O. H.; Magnussen, O. M. A Novel X-Ray Diffractometer for Studies of LiquidLiquid Interfaces. J. Synch. Rad. 2014, 21, 45-56.

(31) Smilgies, D.-M.; Boudet, N.; Struth, B.; Konovalov, O. Troika II: A Versatile Beamline for the Study of Liquid and Solid Interfaces. J. Synch. Rad. 2005, 12, 329-339.

(32) Mars, J.; Hou, B.; Weiss, H.; Li, H.; Konovalov, O.; Festersen, S.; Murphy, B. M.; Rutt, U.; Bier, M.; Mezger, M. Surface Induced Smectic Order in Ionic Liquids - An X-Ray Reflectivity Study of $\left[\mathrm{C}_{22} \mathrm{C}_{1} \mathrm{im}\right]^{+}\left[\mathrm{NTf}_{2}\right]^{-}$. Phys. Chem. Chem. Phys. 2017, 19, 26651-26661, See also Correction note, DOI: 10.1039/c8cp91851a.

(33) Jeon, Y.; Vaknin, D.; Bu, W.; Sung, J.; Ouchi, Y.; Sung, W.; Kim, D. Surface Nanocrystallization of an Ionic Liquid. Phys. Rev. Lett. 2012, 108, 055502. 
(34) Ocko, B. M.; Wu, X. Z.; Sirota, E. B.; Sinha, S. K.; Gang, O.; Deutsch, M. Surface Freezing in Chain Molecules: Normal Nlkanes. Phys. Rev. E 1997, 55, 3164-3182.

(35) Pershan, P. S.; Schlossman, M. L. Liquid Surfaces and Interfaces: Synchrotron X-ray Methods; Cambridge University Press: Cambridge, UK, 2012.

(36) Als-Nielsen, J.; McMorrow, D. Elements of Modern X-ray Physics; Wiely: New York, USA, 2001.

(37) Canongia-Lopes, J. N. A.; Padua, A. A. H. Nanostructural Organization in Ionic Liquids. J. Phys. Chem. B 2006, 110, 3330-3335.

(38) Shimizu, K.; Bernardes, C. E. S.; Canongia Lopes, J. N. Structure and Aggregation in the 1-Alkyl-3-Methylimidazolium Bis(trifluoromethylsulfonyl)imide Ionic Liquid Homologous Series. J. Phys. Chem. B 2014, 118, 567-576.

(39) Araque, J. C.; Hettige, J. J.; Margulis, C. J. Modern Room Temperature Ionic Liquids, a Simple Guide to Understanding Their Structure and How It May Relate to Dynamics. J. Phys. Chem. B 2015, 119, 12727-12740.

(40) Liu, F.; Prehm, M.; Zeng, X.; Tschierske, C.; Ungar, G. Skeletal Cubic, Lamellar, and Ribbon Phases of Bundled Thermotropic Bolapolyphiles. J. Am. Chem. Soc. 2014, $136,6846-6849$.

(41) Als-Nielsen, J.; Birgeneau, R. J.; Kaplan, M.; Litster, J. D.; Safinya, C. R. Experimental Observation of Anomalous Ordering in a Landau-Peierls System. Phys. Rev. Lett. 1977, 39, 1668-1671.

(42) Stamatoff, J.; Cladis, P. E.; Guillon, D.; Cross, M. C.; Bilash, T.; Finn, P. X-Ray Diffraction Intensities of a Smectic- $A$ Liquid Crystal. Phys. Rev. Lett. 1980, 44, 15091512. 
(43) Ocko, B.; Kortan, A.; Birgeneau, R.; Goodby, J. A High Resolution X-Ray Scattering Study of the Phases and Phase Transitions in N-(4-n-Butyloxybenzylidene)-4-nHeptylaniline (40.7). J. Phys. France 1984, 45, 113-128.

(44) Kaganer, V.; Mohwald, H.; Dutta, P. Structure and Phase Transitions in Langmuir Monolayers. Rev. Mod. Phys. 1999, 71, 779-819.

(45) Hernandez Battez, A.; Ramos, D.; Blanco, D.; Gonzalez, R.; Fernandez-Gonzalez, A.; Viesca, J. L. Lubrication Properties of the Ionic Liquid Dodecyl-3 Methylimidazolium bis(trifluoromethylsulfonyl)imide. Tribol. Lett. 2018, 66, 19.

(46) Lipowsky, R. Critical Surface Phenomena at First-Order Bulk Transitions. Phys. Rev. Lett. 1982, 49, 1575-1578.

(47) Yang, Y.; Asta, M.; Laird, B. B. Solid-Liquid Interfacial Premelting. Phys. Rev. Lett. 2013, 110, 096102.

(48) Pott, T.; Méléard, P. New Insight Into the Nanostructure of Ionic Liquids: A Small Angle X-Ray Scattering (SAXS) Study on Liquid Tri-Alkyl-Methyl-Ammonium Bis (Trifluoromethanesulfonyl) Amides and Their Mixtures. Phys. Chem. Chem. Phys. 2009, 11, 5469-5475.

(49) Lee, K.; Lee, C.; Lin, I. B. First Example of Interdigitated U-Shape Benzimidazolium Ionic Liquid Crystals. Chem. Commun. 1997, 899-900.

(50) Nelyubina, Y. V.; Shaplov, A. S.; Lozinskaya, E. I.; Buzin, M. I.; Vygodskii, Y. S. A New Volume-Based Approach for Predicting Thermophysical Behavior of Ionic Liquids and Ionic Liquid Crystals. J. Am. Chem. Soc. 2016, 138, 10076-10079.

(51) Li, T.; Xu, F.; Shi, W. Ionic liquid Crystals Based on 1-Alkyl-3-Methylimidazolium Cations and Perfluorinated Sulfonylimide Anions. Chem. Phys. Lett. 2015, 628, 9-15. 
(52) Kjaer, K.; Als-Nielsen, J.; Helm, C. A.; Tipman-Krayer, P.; Mohwald, H. Synchrotron X-Ray Diffraction and Refelction Studies of Arachidic Acid Monolayers at the AirWater Interface. J. Phys. Chem. 1989, 93, 3200-3206.

(53) Sloutskin, E.; Sapir, Z.; Bain, C. D.; Lei, Q.; Wilkinson, K. M.; Tamam, L.; Deutsch, M.; Ocko, B. M. Wetting, Mixing, and Phase Transitions in Langmuir-Gibbs Films. Phys. Rev. Lett. 2007, 99, 136102.

(54) Yefet, S.; Sloutskin, E.; Tamam, L.; Sapir, Z.; Cohen, A.; Deutsch, M.; Ocko, B. M. Surfactant-Induced Phases in Water-Supported Alkane Monolayers: I. Thermodynamics. Langmuir 2014, 30, 8000-8009.

(55) Yefet, S.; Sloutskin, E.; Tamam, L.; Sapir, Z.; Deutsch, M.; Ocko, B. M. SurfactantInduced Phases in Water-Supported Alkane Monolayers: II. Structure. Langmuir 2014, 30, 8010-8019.

(56) Wilkinson, K. M.; Qunfang, L.; Bain, C. D. Freezing Transitions in Mixed Surfactant/Alkane Monolayers at the Air-Solution Interface. Soft Matter 2006, 2, 66-76.

(57) Matsubara, H.; Takaichi, T.; Takiue, T.; Aratono, M.; Toyoda, A.; Iimura, K.; Ash, P. A.; Bain, C. D. Morphological Transformations in Solid Domains of Alkanes on Surfactant Solutions. J. Phys. Chem. Lett. 2013, 4, 844-848.

(58) Matsubara, H.; Aratono, M. Unique Interfacial Phenomena on Macroscopic and Colloidal Scales Induced by Two-Dimensional Phase Transitions. Langmuir 2019, 35, In press. DOI $=10.1021 /$ acs.langmuir.8b01203.

(59) Bernardini, C.; Stoyanov, S. D.; Cohen Stuart, M. A.; Arnaudov, L. N.; Leermakers, F. A. M. Polymers at the Water/Air Interface, Surface Pressure Isotherms, and Molecularly Detailed Modeling. Langmuir 2010, 26, 11850-11861. 
(60) Lei, Q.; Bain, C. D. Surfactant-Induced Surface Freezing at the Alkane-Water Interface. Phys. Rev. Lett. 2004, 92, 176103.

(61) de Gennes, P. G. Wetting: Statics and Dynamics. Rev. Mod. Phys. 1985, 57, 827-863.

(62) Bonn, D.; Eggers, J.; Indekeu, J.; Meunier, J.; Rolley, E. Wetting and Spreading. Rev. Mod. Phys. 2009, 81, 739-805.

(63) Guinier, A.; Fournet, G. Small-Angle Scattering of X-Rays; Wiley: New York, USA, 1955.

(64) Steinrueck, H.-G.; Will, J.; Magerl, A.; Ocko, B. M. Structure of n-Alkyltrichlorosilane Monolayers on $\mathrm{Si}(100) / \mathrm{SiO}$. Langmuir 2015, 31, 11774-11780.

(65) Haddad, J.; Steinrueck, H.-G.; Hlaing, H.; Kewalramani, S.; Pontoni, D.; Reichert, H.; Murphy, B. M.; Festersen, S.; Runge, B.; Magnussen, O. M. et al. Order and Melting in Self-Assembled Alkanol Monolayers on Amorphous SiO2. J. Phys. Chem. C 2015, $119,17648-17654$.

(66) Ocko, B. M.; Hlaing, H.; Jepsen, P. N.; Kewalramani, S.; Tkachenko, A.; Pontoni, D.; Reichert, H.; Deutsch, M. Unifying Interfacial Self-Assembly and Surface Freezing. Phys. Rev. Lett. 2011, 106, 137801.

(67) Kelton, K. Crystal Nucleation in Liquids and Glasses. Solid State Phys. 1991, 45, $75-177$.

(68) Huber, P. Soft Matter in Hard Confinement: Phase Transition Thermodynamics, Structure, Texture, Diffusion and Flow in Nanoporous Media. J. Phys.-Condes. Matter 2015, $27,103102$.

(69) Penalber-Johnstone, C.; Adamova, G.; Plechkova, N. V.; Bahrami, M.; GhaedSharaf, T.; Ghatee, M. H.; Seddon, K. R.; Baldelli, S. Sum Frequency Generation 
Spectroscopy of Tetraalkylphosphonium Ionic Liquids at the Air-Liquid Interface. $J$. Chem. Phys. 2018, 148, 193841.

(70) Baldelli, S. Surface Structure at the Ionic Liquids Electrified Metal Interface. Acc. Chem. Res. 2008, 41, 421-431. 
Graphical TOC Entry

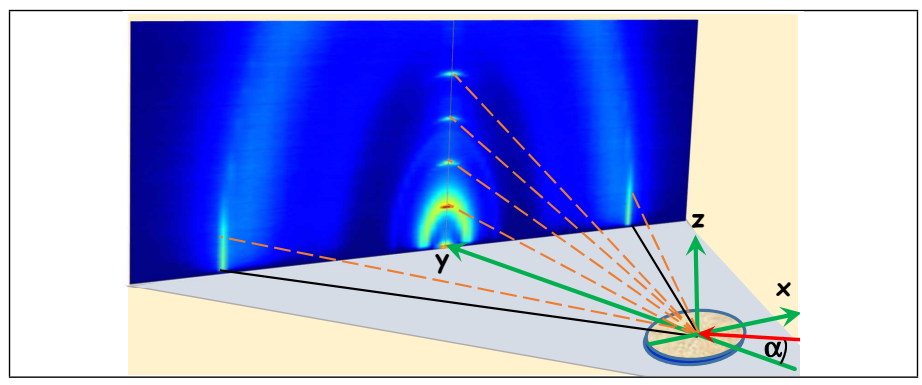

